\title{
DESENVOLVIMENTO DE JOGO DE COMPUTADOR PARA INTERAÇÃO ENTRE UMA PESSOA PORTADORA DE DEFICIÊNCIA VISUAL E OUTRA NÃO PORTADORA
}

\author{
Pablo Ikeda, Mário Augusto Pazoti \\ Faculdade de (FIPP) - Universidade do Oeste Paulista (UNOESTE) - Presidente Prudente - SP. E-mail: mario@unoeste.br
}

\section{RESUMO}

O presente trabalho apresenta o desenvolvimento de um jogo de computador que permite a interação entre dois usuários, sendo um destes portador de deficiência visual, que será guiado por meio de avisos sonoros. De acordo com o censo de 2000 do IBGE, cerca de $10 \%$ da população possui deficiência visual completa ou parcial. Não há disponíveis muitas ferramentas que permitam tal interação. Para o desenvolvimento deste jogo foram utilizadas ferramentas gratuitas como Blender3D e Audacity. $O$ resultado do trabalho desenvolvido foi bastante satisfatório e ressalta o interesse e a necessidade de programas de entretenimento que permitam interação entre pessoas com limitações distintas. Vale ressaltar que um dos maiores desafios na implementação advém do fato de programadores não dispor da sensibilidade auditiva, como os portadores de deficiência visual dispõem, tornando relevante a participação destes durante todo o processo.

Palavras-chave: deficiência visual - Jogo para computador - game engine

\section{DEVELOPING COMPUTER GAME FOR INTERACTION BETWEEN PERSONS WITH AND WITHOUT VISUAL IMPAIRMENT}

\begin{abstract}
This paper presents the development of a computer game that allows interaction between two users, one of them visually impaired, to be guided by sound alerts. According to the IBGE census of 2000, about $10 \%$ of the population has complete or partial visual impairment. There are not many tools available that allow such interaction. To develop this game we have used free tools like Audacity and Blender3D. The result of the work was very satisfactory and highlights the interest and need for entertainment programs that allow interaction between people with distinct limitations. It is noteworthy that one of the greatest challenges in implementation stems from the fact that programmers do not have auditory sensitivity, such as blind people have, making it relevant to their participation throughout the process.
\end{abstract}

Keywords: visual impairment - Computer game - game engine 


\section{INTRODUÇÃO}

Em virtude do apoio dado pelo governo e empresas privadas, visando à inclusão digital, o número de pessoas que possuem acesso à informática no Brasil aumentou, principalmente, nos grandes centros como o estado de São Paulo. Porém, essa acessibilidade não corresponde à totalidade da população, pois parte dela ainda tem poder aquisitivo muito baixo e não dispõe de acessos fáceis, ou possuem alguma limitação física por parte do próprio usuário. Uma pesquisa realizada no ano de 2000 pelo IBGE mostrou que a deficiência visual completa ou parcial é um dos tipos de deficiência que mais atinge a população, cerca de $10 \%$. Com base nisso, pode ser observado que um dos maiores problemas da inclusão digital aos portadores de deficiência visual é a falta ou um pequeno número de aplicações de entretenimento com recursos de acessibilidade. Atualmente existem poucos produtos de entretenimento baseados em computador para pessoas portadoras de deficiência visual com interação e participação de uma pessoa não portadora desse tipo de deficiência. Tais considerações, aplicadas ao desenvolvimento de jogo para portadores de deficiência visual, revelam a necessidade de adaptações e melhorias. $O$ processo de desenvolvimento pode ser o mesmo, porém, deve receber adaptações nos efeitos sonoros e, em alguns casos, é necessário avaliar a acessibilidade em relação aos tipos de controles de acesso.

Essa interação é importante, principalmente, aos deficientes visuais, pois a sensação de igualdade e acessibilidade é maior quando este se vê competindo ou interagindo com outra pessoa no mesmo ambiente virtual. Com este intuito, o presente trabalho teve por objetivo desenvolver um jogo de corrida automobilística que permita a interação entre dois usuários, sendo um desses portadores de deficiência visual, o qual será guiado por meio de avisos sonoros, e outro por meio da interface gráfica. De acordo com Reis Junior (2002), a plataforma na qual o jogo será executado, deve ser previamente definida. Esta se identifica com o hardware, computadores, celulares e o conhecimento das especificidades é imprescindível para que, no final do processo de produção, o jogo seja lançado sem qualquer deficiência.

Visando ao desenvolvimento de um software gratuito, de qualidade e que possa ser adaptado ou remodelado por qualquer programador interessado nesse tipo de estudo, houve a preocupação de utilizar apenas ferramentas livres. Neste trabalho foi utilizado o Blender3D como ferramenta de modelagem o Audacity para a edição de áudio e a programação foi desenvolvida usando o Python, bastante importante para a integração das tecnologias envolvidas.

Mediante os testes efetuados por portador de deficiência visual da entidade assistencial participante deste projeto foi possível testar e aprimorar o jogo. Parte dos testes foi realizada por integrantes da Laramara (Associação Brasileira de Assistência ao Deficiente Visual) e da Associação Filantrópica de Proteção aos Cegos de Presidente Prudente. Os resultados são bastante animadores, visto o entusiasmo dos portadores de deficiência visual quando da utilização do jogo. Além disso, vale destacar que o jogo permite interação entre um usuário portador de deficiência visual e um não portador, os dispositivos de controle correspondem ao almejado e a possibilidade de criação de um único jogo destinado a alguns tipos de usuários é possível sem que haja um aumento excessivo no orçamento do projeto.

Na Seção 2 será apresentada a pesquisa realizada sobre a deficiência visual e os tipos de feedback relacionados à área de jogos. Na Seção 
3 serão discutidos as etapas e os procedimentos para a criação de um jogo. Na Seção 4 serão apresentadas as ferramentas utilizadas. A Seção 5 contém todo o processo de desenvolvimento do jogo. As avaliações e testes são descritos na Seção 6. Na Seção 7 os resultados, as melhorias e as limitações são apresentados, permitindo um maior entendimento de quais as vantagens em criar jogos com esse tipo de acessibilidade. Por fim, são apresentadas a conclusão deste trabalho, as suas contribuições $e$ as possibilidades para se dar continuidade ao projeto.

\section{DEFICIÊNCIA VISUAL}

O termo deficiência visual refere-se a uma situação irreversível de diminuição da resposta visual, em virtude de causas congênitas ou hereditárias, mesmo após tratamento clínico e/ ou cirúrgico e uso de óculos convencionais. A diminuição da resposta visual pode ser leve, moderada, severa, profunda (que compõem 0 grupo de visão subnormal ou baixa visão) e ausência total da resposta visual (cegueira).

De acordo com Laramara (2009),

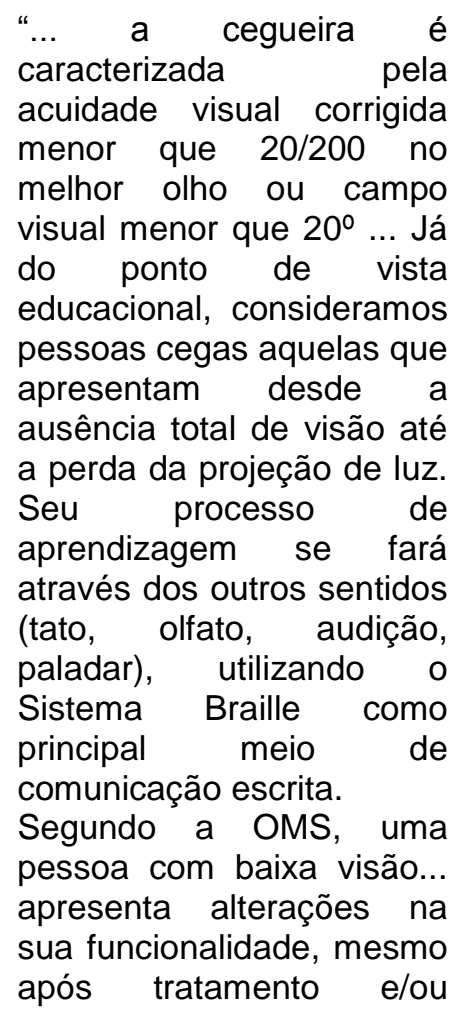

correção óptica, com acuidade visual menor que 20/70 até percepção de luz (sendo a normal equivalente a 20/20); campo visual inferior a $10 \%$ do seu ponto de fixação; alterações na sensibilidade aos contrastes e cores; dificuldade de adaptação à iluminação, mas com capacidade potencial de utilização da visão para o planejamento e execução de tarefas."

Levando em consideração estas informações, é possível notar que existem diferentes graus de deficiência visual e para permitir acessibilidade a esta diversidade em jogos computacionais, é necessário obter conhecimento dos diversos dispositivos de interação entre usuário e software, e quais as reações ou retorno (feedback) que o jogo emitirá. Outro fator importante que deve ser ressaltado é - aumento da capacidade auditiva e da orientação espacial por pessoas com tal deficiência. Esta afirmação está embasada na Neurociência e Psicologia, áreas que estudam a complementação por parte do próprio organismo, o qual é forçado a fazer isso, em caso da perda de algum sentido humano. De acordo com Sá et al (2007) "sem a visão, os outros sentidos passam a receber a informação de forma intermitente, fugidia e fragmentária. desenvolvimento aguçado da audição, do tato, do olfato e do paladar é resultante da ativação contínua desses sentidos por força da necessidade".

\section{Feedback}

De acordo com Barfield (1993 apud Carvalho, 1994, p. 53), o vocábulo feedback diz respeito a uma comunicação de informação ao usuário e esse retorno proporciona as informações a respeito de sua interação com o jogo, auxiliandoo na correta execução de seu intento, tornando- 
se, então, um dos ingredientes vitais para a interação usuário-sistema.

Levando em consideração que o projeto todo é voltado a deficientes visuais e aos não deficientes visuais, alguns retornos são extremamente necessários, em especial os auditivos. O feedback visual é a forma mais importante e amplamente utilizada de canal em sistemas interativos de computador, sendo que a tela dos monitores de vídeo é o principal alvo deste canal, apesar de existirem outras formas de retorno visual. $\mathrm{O}$ feedback auditivo é também amplamente utilizado, porém, ele não pode proporcionar a mesma riqueza de informação quando comparada àquela proporcionada pelo feedback visual, mas é talvez o único que permita a interação com um deficiente visual.

Além das características técnicas, é preciso definir também as questões de acessibilidade que possibilite melhor interação entre pessoas portadoras e não portadoras de deficiência visual. De acordo com Jan Hunt (2006), as crianças aprendem a se relacionar melhor, convivendo com pessoas de todas as idades e o uso do computador aumenta a possibilidade desse relacionamento e, de acordo com Oliveira (2006), as crianças apresentam intensa motivação para o conhecimento e o manuseio de computadores, jogos e agendas eletrônicas. Os estudos de Oliveira (2006) e Hunt (2006) demonstram que a oportunidade de interação virtual permite o envolvimento de pessoas com necessidades especiais distintas, porém, com o mesmo propósito.

Dessa forma, jogos infantis com objetivo educacional podem ser utilizados com o intuito de proporcionar o aprendizado e a interação entre crianças, aumentando a integração/interação logo no início das atividades infantis. Outro fato importante é a possibilidade de um professor ou orientador, utilizar um jogo para educar, podendo acompanhar visualmente o jogo e ensinar uma criança a usar teclado, o mouse e até mesmo orientar a criança quanto às funcionalidades quando ela estiver jogando.

\section{JOGO DE COMPUTADOR}

Todo jogo de computador consiste em um conjunto de artes distintas, que ao serem unidas e sincronizadas formam uma só. A ideia de arte é subjetiva, portanto, não poderá agradar a todos. As definições para a elaboração de um bom jogo, segundo Casey O'Donnell (2009), são Conceito, Desenvolvimento, Avaliação e Finalização. Cada etapa possui uma ou mais artes, portanto, para que seja possível concluir o projeto será necessário o mínimo de conhecimento em todas estas artes ou aliar-se a pessoas que tenham tal habilidade. Segue o detalhamento de cada uma dessas fases:

a) Conceito: uma ideia original, ou uma adaptação de obras cinematográficas ou livros pode ser referência para a criação de um jogo. Inicialmente são definidos os aspectos técnicos, como formato do produto final e equipamentos para os quais ele será lançado, permitindo assim, definir o público alvo e as necessidades físicas para que a aplicação tenha êxito. Em seguida, e necessário se definir o tema, o estilo, a ambientação e os personagens principais. Artistas e roteiristas esboçam ilustrações e imagens conceituais, que revelam as impressões iniciais do projeto (IGDA, 2009).

b) Desenvolvimento: nessa etapa, o jogo começa a ser criado, reunindo roteiro, programação e direção artística. A união e o sincronismo das artes são totalmente colocados em prática nessa fase e isso é possível com a habilidade e conhecimento de um programador, o qual implementa a parte lógica do jogo integrando todas as demais artes já elaboradas para o projeto. Nesse projeto, em específico, toda implementação lógica baseou-se na ferramenta Blender3D juntamente com scripts Python. 
c) Avaliação: nessa etapa já se têm os protótipos, ou versões parciais do projeto, a partir das quais são realizados testes para o aprimoramento de aspectos específicos do jogo como: combate ou disputa, movimentação, utilização de controles, condução de veículos, colisão de objetos, jogabilidade, entre outros. Deve-se apurar os defeitos e corrigi-los a cada versão de teste que for lançada. Essas versões são criadas para os jogadores até que retorne uma avaliação que não apresente erros que comprometam o jogo, então, o projeto é declarado concluído (IGDA, 2009).

d) Finalização: a partir do lançamento, o jogo pode ganhar atualizações para corrigir novos erros descobertos ou pacotes de expansão com conteúdo adicional. As expansões devem ser premeditadas, assim a equipe do projeto terá estrutura para futuras melhorias com maior facilidade e menor número de erros e problemas. No caso da descoberta de erros, toda a equipe deverá trabalhar para encontrar e solucionar, porém, deve existir um plano de ação para tais possibilidades de erro.

\section{FERRAMENTAS}

Visando ao desenvolvimento de um software gratuito, de qualidade e que possa ser adaptado ou remodelado por qualquer programador interessado nesse tipo de estudo, foram utilizadas apenas ferramentas livres, que demandam do desenvolvedor apenas treinamento ou conhecimento das mesmas, informações que podem ser adquiridos em materiais disponibilizados em bibliotecas ou sites. O resultado em tempo hábil e positivo do trabalho foi possível devido à escolha de ferramentas que são abordadas nas subseções abaixo.

\section{Blender 3D}

Blender 3D é um programa de computador de código aberto, desenvolvido pela Blender Foundation, para modelagem, animação, texturização, composição, renderização, edição de vídeo e criação de aplicações interativas em 3D através de seu motor de jogo integrado, o Blender Game Engine. O programa é multiplataforma, portanto, disponível para diversos sistemas operacionais (Blender, 2010).

O Blender implementa avançadas ferramentas de simulação, tais como: dinâmica de corpo rígido, dinâmica de corpo macio e de fluido; avançadas ferramentas de modelagem; ferramentas de animação de personagens, sistema de materiais baseados em nós e composição de imagens e vídeos. Inclui suporte a Python como linguagem de script, que pode ser usada tanto no Blender, quanto em seu motor de jogo.

Para a construção dos objetos, foram aplicados conceitos de modelagem 3D (Roger, 1997). A modelagem 3D de um jogo foi dividida em três fases: texturização, atribuição de ação e som aos objetos. Cada uma dessas fases possui etapas mais específicas e que serão abordadas nas seções a seguir. Na Figura 1 é ilustrada a modelagem 3D parcialmente concluída de um dos objetos deste projeto.

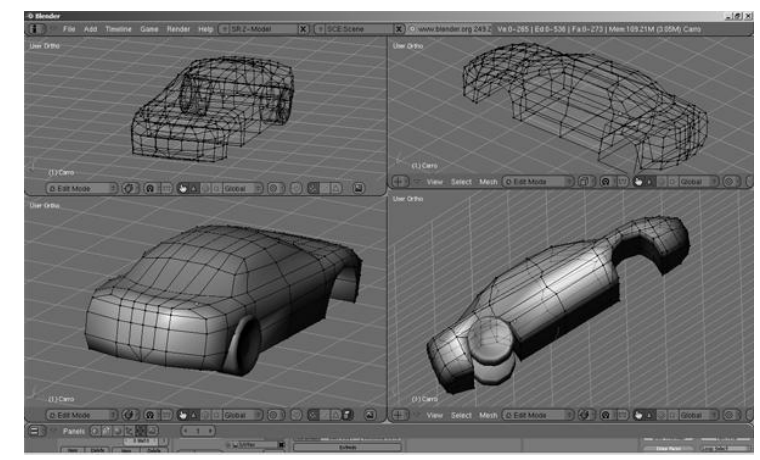

Figura 1. Objeto Tridimensional Blender3D Veículo do jogo

\section{Audacity}

A ferramenta Audacity permite modificar, adicionar efeitos, realizar cortes, entre outros, porém, de áudios prontos. Inicialmente, para o projeto, as falas foram geradas através da ferramenta TEXT TO WAV, que consiste no 
pacote SAPi5, em que a frase ou a palavra é digitada e o programa gera o respectivo áudio. Entretanto, por motivos legais, as falas foram regravadas com microfone e substituídas no projeto final. A tecnologia de edição de áudio é fundamental para o desenvolvimento de jogos para portadores de deficiência visual, ela permite adequar todos os efeitos e falas atingindo com maior ênfase o objetivo proposto.

\section{DESENVOLVIMENTO DO TRABALHO}

Embasado em todo material e ferramentas pesquisadas, o trabalho teve início de acordo com as quatro etapas de criação de um jogo. Relatos de portadores de deficiência visual em conjunto com não portadores definiram a escolha do tipo de jogo - Corrida Automobilística. Assim, esboços dos menus de acesso e estilo de cenário foram desenvolvidos visando a atender ao conceito de criação de um jogo. Na Figura 2 são mostrados dois rascunhos elaborados para a concepção do menu e, parcialmente, o cenário da primeira fase do jogo.

O tipo de voz que irá transmitir as informações foi determinado nessa etapa, no entanto, as falas são criadas após o desenvolvimento gráfico, ou seja, depois de definida, modelada e estruturada a fase do jogo.

Com os rascunhos digitalizados, a etapa de desenvolvimento iniciou-se utilizando a ferramenta Blender3D em modo de edição de objeto. Os menus de acesso começam a ser gerados, integrando as ações e animações aos objetos por meio de controles lógicos existentes na ferramenta (sensores, controladores e atuadores). Variáveis dos tipos inteiro e lógica são definidas, permitindo contagem e validação da escolha a um tipo de seleção do menu, entre outras tarefas.

Todo o processo de desenvolvimento para os menus internos consiste numa estrutura simples com até quatro opções, com modificação apenas no tipo de opção e da respectiva fala. No total, são quatro menus, sendo eles: Principal, Um jogador (Figura 3a), Dois jogadores (Figura 3b) e Opções (Figura 3c). Quando escolhida a opção para "Dois jogadores", são mostradas as duas câmeras do jogo (dividindo-se a tela). Entretanto, é possível selecionar a opção "Somente áudio", na qual a parte gráfica usada pelo deficiente visual fica oculta.

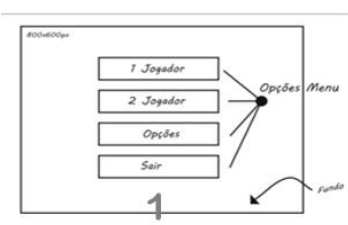

Figura 2.

$$
\begin{gathered}
\text { Croqui do } \\
\text { menu } \\
\text { principal (1) } \\
\text { e cenário (2) }
\end{gathered}
$$

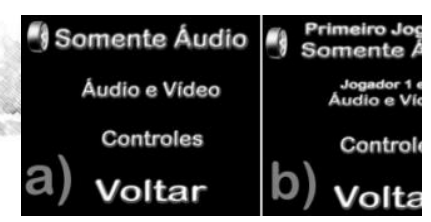

Figura 3.
Menu: Um

jogador (a),

Dois

Jogadores

(b) $\mathrm{e}$

Opções (c)

Concretizada toda a etapa de acesso, as avaliações e os testes começaram a ser realizados por portadores da deficiência visual, pois são eles os principais usuários do jogo. Os portadores de deficiência visual, como já mencionado, possuem acuidade auditiva superior a uma pessoa não portadora de deficiência visual e uma orientação espacial bastante desenvolvida e, com isso, suas observações auxiliam bastante no desenvolvimento e adequação do jogo a suas necessidades.

Após o teste dos menus e a aprovação pelos usuários, iniciou-se o desenvolvimento dos veículos, sensores, cenários e controles de acesso. Para essa etapa, texturas de fundo, como montanhas e céu foram obtidas em sites gratuitos (http://www.cgtextures.com/). Para o jogo foram desenvolvidos objetos 3D como: veículos, pilares, pista, árvores, ou seja, todo conteúdo de cada fase. Em seguida, foram aplicadas as texturas e 
os sensores de colisão foram definidos e implementados. Por fim, foram definidas as teclas a serem utilizadas para interação no jogo e também os botões do joystick. As teclas, definidas par ao jogo com o auxílio de um deficiente visual, são mostradas na Tabela 1.

Tabela 1. Teclas, botões em suas funcionalidades

\begin{tabular}{|c|c|c|c|}
\hline $\begin{array}{l}\text { Opções de } \\
\text { jogador }\end{array}$ & Dispositivo & Tecla ou Botão & Ação \\
\hline \multirow{12}{*}{$\begin{array}{l}\text { Primeiro } \\
\text { jogador }\end{array}$} & Teclado & Seta para cima & \multirow{2}{*}{ Acelerar } \\
\hline & Joystick & Botão Quadrado & \\
\hline & Teclado & Seta para baixo & \multirow{2}{*}{ Frear } \\
\hline & Joystick & Botão X & \\
\hline & Teclado & Seta para direita & \multirow{2}{*}{ Virar à direita } \\
\hline & Joystick & Direcional para lado direito & \\
\hline & Teclado & Seta para esquerda & \multirow{2}{*}{ Virar à esquerda } \\
\hline & Joystick & Direcional para lado esquerdo & \\
\hline & Teclado & CTRL lado direito & \multirow{2}{*}{ Buzinar } \\
\hline & Joystick & $\mathrm{R} 1$ & \\
\hline & Teclado & Enter & \multirow{2}{*}{$\begin{array}{l}\text { Acessar os menus e } \\
\text { durante o jogo Pausar }\end{array}$} \\
\hline & Joystick & Start & \\
\hline \multirow{12}{*}{$\begin{array}{l}\text { Segundo } \\
\text { jogador }\end{array}$} & Teclado & W & \multirow{2}{*}{ Acelerar } \\
\hline & Joystick & Botão Quadrado & \\
\hline & Teclado & $\mathrm{S}$ & \multirow{2}{*}{ Frear } \\
\hline & Joystick & Botão X & \\
\hline & Teclado & $\mathrm{D}$ & \multirow{2}{*}{ Virar à direita } \\
\hline & Joystick & Direcional para lado direito & \\
\hline & Teclado & A & \multirow{2}{*}{ Virar à esquerda } \\
\hline & Joystick & Direcional para lado esquerdo & \\
\hline & Teclado & CTRL lado esquerdo & \multirow{2}{*}{ Buzinar } \\
\hline & Joystick & $\mathrm{R} 1$ & \\
\hline & Teclado & Enter & \multirow{2}{*}{$\begin{array}{l}\text { Acessar os menus e } \\
\text { durante o jogo Pausar }\end{array}$} \\
\hline & Joystick & Start & \\
\hline
\end{tabular}

Finalizados os objetos $3 \mathrm{D}$ e as variáveis de controle, foram definidos outros itens é como: a quantidade de veículos em pista, a quantidade de voltas para cada um deles, o controle de velocidade e a colisão. Para cada um desses controles, foram acopladas informações sonoras e visuais, atendendo aos critérios de acessibilidade. Algumas adequações exigem maior empenho por parte do programador, como por exemplo, o controle de marcha do veículo, que é mostrado em tela por meio de um número, mas que para os deficientes visuais a alternativa foi distinguir pelo som do torque do motor.

É importante ressaltar que todas as informações necessárias para que um deficiente visual possa jogar foram minuciosamente testadas e aprovadas. As falas em um jogo para esse público devem ser curtas e diretas e os efeitos sonoros e bips devem ser emitidos no momento exato. Na Figura 4 são mostradas as quatro etapas para o desenvolvimento do jogo efetuadas na ferramenta Blender3D. 


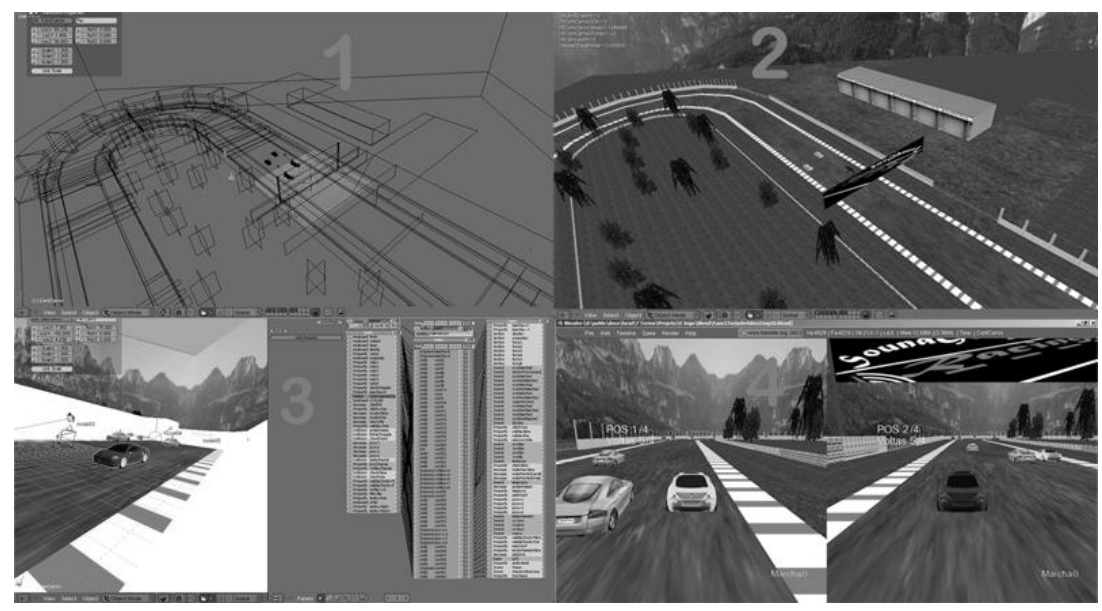

Figura 4. llustração das quatro etapas para o desenvolvimento: 1) Conceito, 2) Desenvolvimento, 3) Avaliação, 4) Finalização

A realização das etapas no desenvolvimento de jogos foi bastante trabalhosa, principalmente, quando há a necessidade de emissão de falas e efeitos sonoros no tempo exato. Para possibilitar isso, todo o material sonoro foi equalizado e modificado com a ferramenta Audacity, por meio da qual foi possível aumentar o volume em determinada faixa de tempo e frequência, realizar cortes e, principalmente, aumentar a velocidade da fala sem perdas perceptíveis.

A sincronização, a adaptação e a integração dessas tecnologias foram possíveis através da linguagem de programação Python. A ferramenta Blender3D possui um motor de jogos que usa scripts em seus controladores, atuadores e sensores, de forma a permitir ainda que scripts personalizados sejam adicionados. Para 0 desenvolvimento desse trabalho poucos scripts personalizados foram utilizados. Um deles é o que permite a divisão da tela sem prejuízo ao processamento de imagem, levando em consideração a necessidade de processamento duplo quando há dois jogadores.

O game engine e seus componentes são configurados e preparados para a realização de várias atividades por meio de Threads, que são gerenciadas automaticamente pelo motor de jogo.
Existe a possibilidade de se criar e gerenciar, porém, é uma tarefa um tanto quanto complexa.

Elaborado todo processo de desenvolvimento do jogo, são necessários os testes de jogabilidade, sendo que isso foi primeiro realizado pelo desenvolvedor, com intuito de resolver problemas decorrentes da implementação, como problemas de colisão, efeitos sonoros fora de sincronismo, entre outros, e depois foi encaminhado para avaliação de um portador de deficiência visual, para se avaliar a jogabilidade.

\section{AVALIAÇÃO DO JOGO POR PORTADORES DE DEFICIÊNCIA VISUAL}

Durante todo 0 desenvolvimento, avaliações constantes foram necessárias para garantir a funcionalidade do software, sendo considerados testes com relação às técnicas utilizadas (programação) e de jogabilidade. Os testes foram efetuados usando tanto o teclado quanto o joystick. As orientações dadas pelo deficiente visual da Laramara auxiliaram na definição de quais teclas deveriam ser utilizadas, bem como solicitou o acréscimo de recursos aos veículos, como por exemplo, o som de buzina, que são detalhes que passam despercebidos por uma pessoa que não possui a limitação visual. 
As orientações e diálogos foram realizados por meio de e-mails e o jogo foi disponibilizado em um servidor para que fosse possível o download e a instalação do mesmo na instituição. A seguir são mostrados alguns trechos recortados de e-mail enviados pelo deficiente visual.

"Vasculhei todo o menu e
está operando conforme o
esperado, só que a tecla
para acelerar você devia
colocar como seta para
cima e freio seta para
baixo."
"Esquerda e direita seta
esquerda e direita. Coloque
também uma opção de
buzina."
"A forma de jogar via
teclado ficou muito boa, a
buzina próxima dos
controles e tudo...RS."

O programa foi considerado funcional e bastante satisfatório para o uso de pessoas com deficiência visual, auditiva ou sem deficiência.

\section{RESULTADOS}

Visando ao desenvolvimento de um software acessível, de qualidade e que possa ser adaptado ou remodelado por qualquer programador interessado nesse tipo de estudo, as ferramentas gratuitas utilizadas atenderam em todos os aspectos projetados, garantindo a possível adaptação ou remodelagem por outro programador, demandando deste apenas treinamento ou conhecimento das técnicas utilizadas.

Os testes e a avaliação por portador de deficiência visual em toda etapa do desenvolvimento foram fundamentais para a obtenção de um resultado satisfatório, pois são eles os usuários para os quais foi desenvolvido o software. Essas avaliações permitiram aprimorar o jogo e os resultados são animadores, visto o entusiasmo dos avaliadores e portadores da deficiência visual.
Além de entretenimento, o jogo possibilita que um orientador ou professor acompanhe o desenvolvimento do seu aluno quando da utilização do jogo, pois, com a parte gráfica do jogo é garantido que o portador de deficiência seja orientado tanto pelos sensores do jogo quanto por um instrutor, pelo menos no início. $\mathrm{O}$ aprendizado e o entretenimento baseados em interação, acessibilidade e integração são vistos em todos os aspectos do jogo, sendo que as possibilidades de interação não se limitam apenas entre o deficiente visual e computador. $\mathrm{O}$ mesmo pode jogar com uma pessoa com deficiência auditiva, ou com uma pessoa sem deficiências.

É bastante difícil que um programador ou uma equipe desenvolva um software com esse propósito sem avaliação e testes por parte de um portador de deficiência visual, ou que somente os projetos já existentes forneçam conhecimento suficiente para o tal. Outro ponto importante é que jogos com grande quantidade de detalhes, fases e outros recursos, devem ser elaborados por uma equipe para atingir o objetivo por completo, caso contrário, o tempo para implantação será aumentado, o que pode tornar o jogo defasado ou com grande número de erros, se feito às pressas apenas para cumprir o cronograma estabelecido.

\section{CONCLUSÃO}

Este trabalho permite não somente a apresentação de referenciais para projetistas e usuários de interfaces de computadores destinadas aos deficientes visuais, mas, também, uma visão dos problemas envolvidos na elaboração de jogo de computador com possibilidade de interação entre portador de deficiência visual e não portador. A principal contribuição é a de apresentar um conjunto de regras e etapas para atender as necessidades do portador de deficiência visual em jogos de computador e apresentar ferramentas 
computacionais gratuitas que permitam elaborar todo o projeto.

A partir desse trabalho pôde ser demonstrada a realização de interação virtual entre portadores de deficiência visual e não portador em um mesmo ambiente, desde que, o projeto mantenha um padrão de acesso e todos os meios de comunicação entre usuário e computador seja emitido sonoramente e visualmente, permitindo assim, a acessibilidade ideal para mais de um tipo de usuário. A oportunidade de interação virtual permitiu 0 envolvimento de pessoas com necessidades especiais distintas, porém, com o mesmo propósito, desta forma, jogos infantis com objetivo educacional podem ser utilizados atingindo 0 propósito de aprendizado e interação entre crianças reduzindo o preconceito logo no início das atividades infantis.

Conclui-se que é importante verificar as características de usuários e componentes físicos para a elaboração de um jogo, estudos sobre deficiência visual é imprescindível para todo o desenvolvimento. Apesar de existirem jogos destinados a deficientes visuais, poucos permitem interação entre portador e não portador de deficiência visual em muitos casos. Pessoas denominadas normais sentem a mesma dificuldade em interagir com jogos destinados a deficientes visuais, pois o sentido audição de uma pessoa normal não atinge o grau de sensibilidade de uma pessoa portadora de deficiência visual, além de que, um jogo para deficientes visuais muitas vezes acaba não sendo tão atrativo para outros tipos de usuário quando não disponibiliza ilustrações gráficas.

Este trabalho contribui e demonstra que é possível elaborar software de entretenimento com interação entre pessoas com limitações distintas, dentre elas, deficiência visual, auditiva e pessoas sem necessidades especiais, permitindo assim, ampla inclusão virtual e com acesso a recursos pouco visados pelas empresas deste ramo de desenvolvimento.

\section{REFERÊNCIAS}

Audacity. Disponível em: http://audacity.sourceforge.net/. 2010.

BLENDER. Blender Foundation. Disponível em: http://blender.org. Acessado em: Março 2010

CARVALHO, J. O. F. (1994) Referenciais para projetistas e usuários de interfaces de computadores destinadas aos deficientes visuais. Dissertacão (Mestrado em Engenharia Elétrica) 176 pág. Universidade Estadual de Campinas, Campinas.

HUNT, J. The Natural Child Project. Disponível em: http://www.naturalchild.org/. Acessado em: 3 e 4.Ago. 2010.

IBGE. Instituto Brasileiro de Geografia e Estatística. Brasil. Disponível em: http://www.ibge.gov.br/home/, Acessado em Fev. 2010

IGDA. International Game Developer Association. Disponível em: http://www.igda.org/accessibility. Acesso em: 3 e 4. Ago. 2010

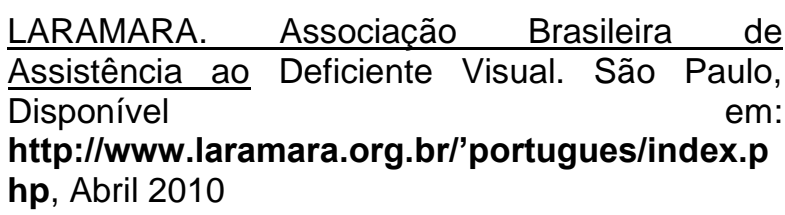

OLIVEIRA, E. S. G. Criança e Computador: Interação que impulsiona o desenvolvimento e a aprendizagem. (2006). 10 f. Artigo - Faculdade de Educação, Universidade do Estado do Rio de Janeiro, Rio de Janeiro.

REIS JUNIOR A. S, NASSU, B.T, JONACK, M. A. Um Estudo Sobre os Processos de Desenvolvimento de Jogos Eletrônicos (Games). 2002. 29.f. Disponível em: http://www.ademar.org/texts/processo-desenvgames.pdf. Acessado em 10/08/2010.

ROGERS, D. F. (1997) Procedural Elements of Computer Graphics. McGraw-Hill Science/Engineering/Math.

SÁ, Elizabet Dias, CAMPOS, Izilda Maria, SILVA, Myriam B. C. Atendimento Educacional Especializado. Brasília. DF, (2007). Disponível em:

http://portal.mec.gov.br/seesp/arquivos/pdf/ae e dv.pdf. Acessado em 10/08/2010. 